J. DIFFERENTIAL GEOMETRY

96 (2014) 223-240

\title{
MANIFOLDS WITHOUT CONJUGATE POINTS AND THEIR FUNDAMENTAL GROUPS
}

\author{
Sergei Ivanov \& Vitali Kapovitch
}

\begin{abstract}
We show that in the fundamental groups of closed manifolds without conjugate points, centralizers of all elements virtually split.
\end{abstract}

\section{Introduction}

It is a classical consequence of Rauch comparison that manifolds of nonpositive curvature have no conjugate points. While the converse need not hold even for closed manifolds [11], the following question remains unanswered in dimensions above 2 .

Question 1.1. Does every closed Riemannian manifold without conjugate points admit a metric of nonpositive curvature?

While the answer to this is likely negative, it has been shown that fundamental groups of closed manifolds without conjugate points satisfy many properties that are known to hold for nonpositively curved manifolds. In particular, Croke and Schroeder [8] proved that if a closed manifold $\bar{M}$ admits an analytic metric without conjugate points then every abelian subgroup of $\pi_{1}(\bar{M})$ is straight (i.e., quasi-isometrically embedded in $\pi_{1}(\bar{M})$ ) and every solvable subgroup of $\pi_{1}(\bar{M})$ is virtually abelian. Both of these properties are known to hold for groups that act isometrically, properly, and co-compactly on CAT(0)-spaces (we refer to such groups as CAT(0)-groups) or, more generally, for the semihyperbolic groups (which is a strictly bigger class of groups; see e.g., [1] or [5] for the definition). Our main result is that the following known property of $\operatorname{CAT}(0)$-groups (see e.g., [5, Theorem 6.12]) also holds for fundamental groups of closed manifolds without conjugate points.

Theorem A. Let $\bar{M}$ be a closed manifold that admits a $C^{\infty}$ Riemannian metric without conjugate points. Then for every nontrivial element $\gamma \in \pi_{1}(\bar{M})$, its centralizer $Z(\gamma)<\pi_{1}(\bar{M})$ virtually splits over $\gamma$.

This means that there exists a finite index subgroup $G<Z(\gamma)$ that is isomorphic to a direct product $\mathbb{Z} \times G^{\prime}$ so that $\gamma$ corresponds to the generator of the $\mathbb{Z}$ factor.

Received 5/23/2012. 
Actually, we prove an equivalent but more convenient property of the centralizer (Corollary 4.3): the image of $\gamma$ in $H_{1}(Z(\gamma))=Z(\gamma) /[Z(\gamma), Z(\gamma)]$ is a non-torsion element.

There are examples of manifolds that can be shown not to admit metrics without conjugate points by Theorem A but not by previously known results. We construct such examples in Section 5 .

The proof of Theorem A builds upon the already mentioned work of Croke and Schroeder $[\mathbf{8}]$. The main result of $[\mathbf{8}]$ (i.e., the straightness of abelian subgroups) by itself does not imply the assertion of Theorem A, but some intermediate results in $[\mathbf{8}]$ do. We remove the analyticity assumption from one of those results (see Proposition 3.1 below) and deduce Theorem A from it.

REMARK 1.2. Kleiner (unpublished) and Lebedeva [14] found simpler proofs of the main result of $[\mathbf{8}]$ that work without analyticity. These proofs go via a different route that does not yield Theorem A.

REMARK 1.3. Our proof requires $C^{\infty}$ regularity of the metric or, more precisely, $C^{r}$ regularity for some $r$ depending on $n=\operatorname{dim} M$. This is needed in Lemma 3.3. We do not know whether Theorem A is true for $C^{r}$ metrics for any fixed $r$.

Examples constructed in [6] show that Lemma 3.3 and an analogue of Proposition 3.1 (in a similar but not identical context) fail if $n \gg r$. However, it might be possible that some features of metrics without conjugate points (e.g., the fact that displacement functions do not have critical points other than minima) can be used to squeeze more regularity out of the problem.

REMARK 1.4. A straightforward modification of the proof shows that Theorem A holds for Finsler metrics as well, but we do not bother the reader with this generalization.

Organization of the paper. In Section 2 we provide necessary background material on the properties of universal covers of closed manifolds without conjugate points. In Section 3 we prove the key technical result Proposition 3.1, which allows us to remove the analyticity assumption from the arguments in [8]. In Section 4 we prove Theorem A. In Section 5 we construct new examples of manifolds not admitting metrics without conjugate points. In Section 6 we prove that fundamental groups of closed manifolds without conjugate points have solvable word and conjugacy problems (Theorem 6.1). In Section 7 we consider an analogue of Question 1.1 for metrics without focal points and prove that the answer is affirmative in dimension 3 (Theorem 7.1).

Lastly, in Section 8 we discuss a number of open problems concerning manifolds without conjugate points.

Acknowledgments. The first author is grateful to the organizers of the "Geometry in Inverse Problems" program held in March-April 2012 at 
Fields Institute, Toronto, where this work started. The second author is very grateful to Misha Kapovich and Ilya Kapovich for many helpful conversations and suggestions during the preparation of this paper.

The first author was supported in part by RFBR grant 11-01-00302a. The second author was supported in part by a Discovery grant from NSERC.

\section{Preliminaries}

In this section we collect the necessary preliminaries, borrowed mainly from $[8]$.

Let $\bar{M}$ be a compact Riemannian manifold without conjugate points and $M$ its universal cover. We represent the fundamental group $\pi_{1}(\bar{M})$ as the group $\Gamma$ of deck transformations of $M$. This group acts on $M$ by isometries and $\bar{M}=M / \Gamma$. We fix the notation $\bar{M}, M$, and $\Gamma$ for the rest of the paper.

Since the metric has no conjugate points, every geodesic in $M$ is minimizing, $\exp _{x}: T_{x} M \rightarrow M$ is a diffeomorphism for every $x \in M$, and the Riemannian distance function $d: M \times M \rightarrow \mathbb{R}_{+}$is smooth outside the diagonal. All geodesics throughout the paper are parametrized by arc length.

Fix a nontrivial element $\gamma \in \Gamma$. The displacement function $d_{\gamma}: M \rightarrow$ $\mathbb{R}_{+}$is defined by

$$
d_{\gamma}(x)=d(x, \gamma x), \quad x \in M .
$$

A complete geodesic $c: \mathbb{R} \rightarrow M$ is called an axis of $\gamma$ if $\gamma$ translates $c$ forward along itself, i.e., there is a constant $L>0$ such that $\gamma c(t)=$ $c(t+L)$ for all $t \in \mathbb{R}$. Note that if $c$ is an axis of $\gamma$, then the reverse geodesic $t \mapsto c(-t)$ is an axis of $\gamma^{-1}$. Let $A_{\gamma} \subset M$ denote the union of all axes of $\gamma$.

The following lemma summarizes several results from $[\mathbf{8}]$ that we will need in what follows.

Lemma 2.1. In the above notation, the following holds.

1) The function $d_{\gamma}$ assumes a positive minimum, $\min d_{\gamma}$. The set of points $x \in M$ where $d_{\gamma}(x)=\min d_{\gamma}$, is equal to $A_{\gamma}$.

2) The isometry $\gamma$ translates all its axes by the same amount, namely $\min d_{\gamma}$. That is, if $c$ is an axis of $\gamma$ then $\gamma(c(t))=c\left(t+\min d_{\gamma}\right)$ for all $t \in \mathbb{R}$.

3) $\min d_{\gamma^{m}}=m \cdot \min d_{\gamma}$ for every integer $m \geq 1$.

4) $A_{\gamma}$ is equal to the set of critical points of $d_{\gamma}$. In particular $d_{\gamma}$ has no critical points outside its minimum set.

Proof. See [8, Lemma 2.1] and remarks there. 
The following lemma is proved in [8, Lemma 2.4], however it is not made very clear there that the proof does not depend on analyticity of the metric. Therefore we include a proof (essentially the same one) here.

Lemma 2.2. The set $A_{\gamma}$ is connected.

Proof. Consider the centralizer $Z(\gamma) \subset \Gamma$ of $\gamma$. It is easy to see that the action of $Z(\gamma)$ on $M$ preserves $d_{\gamma}$, hence $d_{\gamma}$ induces a well defined function $\bar{d}_{\gamma}$ on $M / Z(\gamma)$. Furthermore, $\bar{d}_{\gamma}$ is proper (see [8, Lemma 2.2]) and by Lemma 2.1(4) it has no critical point outsize its minimum set $\bar{A}_{\gamma}$. Therefore by Morse theory every sublevel set

$$
\bar{U}_{\varepsilon}=\left\{x \in M / Z(\gamma): \bar{d}_{\gamma}(x) \leq \min \bar{d}_{\gamma}+\varepsilon\right\}, \quad \varepsilon>0,
$$

is a strong deformation retract of $M / Z(\gamma)$. Since the projection $\pi: M \rightarrow$ $M / Z(\gamma)$ is a covering map, it follows that the set

$$
U_{\varepsilon}:=\pi^{-1}\left(\bar{U}_{\varepsilon}\right)=\left\{x \in M: d_{\gamma}(x) \leq \min d_{\gamma}+\varepsilon\right\}
$$

is a strong deformation retract of $M$. Hence $U_{\varepsilon}$ is connected. Since $A_{\gamma}=\bigcap_{\varepsilon>0} U_{\varepsilon}$, it follows that $A_{\gamma}$ is connected as well. $\quad$ q.e.d.

REMARK 2.3. The proof of Lemma 2.2 implies that $Z(\gamma)$ is finitely generated and finitely presented since it shows that $M / Z(\gamma)$ is homotopy equivalent to $\bar{U}_{\varepsilon}$, which is a compact manifold with boundary.

The Busemann function of a (minimizing) geodesic $c: \mathbb{R} \rightarrow M$ is a function $b_{c}: M \rightarrow \mathbb{R}$ defined by

$$
b_{c}(x)=\lim _{t \rightarrow+\infty} d(x, c(t))-t .
$$

The triangle inequality implies that the function $t \mapsto d(x, c(t))-t$ is non-increasing, hence $b_{c}(x)$ is well defined and $b_{c}(x) \leq d(x, c(t))-t$ for all $t \in \mathbb{R}$. Note that $b_{c}(c(t))=-t$ for all $t \in \mathbb{R}$.

Clearly Busemann functions are 1-Lipschitz. Busemann functions are naturally translated by isometries; namely, if $\alpha: M \rightarrow M$ is an isometry then $b_{c}(x)=b_{\alpha c}(\alpha x)$ for all $x \in M$. Changing the origin of a geodesic adds a constant to its Busemann function; namely, if $c_{1}(t)=c(t+L)$, where $L$ is a constant, then $b_{c_{1}}(x)=b_{c}(x)+L$.

Lemma 2.4. Let $c$ and $c_{1}$ be axes of $\gamma$. Then

1) $b_{c}(\gamma x)=b_{c}(x)-\min d_{\gamma}$ for all $x \in M$.

2) $b_{c}$ decays at unit rate along $c_{1}$, that is $b_{c}\left(c_{1}\left(t+t_{1}\right)\right)=b_{c}\left(c_{1}(t)\right)-t_{1}$ for all $t, t_{1} \in \mathbb{R}$.

Proof. Let $L=\min d_{\gamma}$. Then $b_{c}(\gamma x)=b_{\gamma^{-1} c}(x)=b_{c}(x)-L$ since $\gamma^{-1} c(t)=c(t-L)$ by Lemma 2.1(2). This proves the first assertion. It implies that

$$
b_{c}\left(c_{1}(t+m L)\right)=b_{c}\left(\gamma^{m} c_{1}(t)\right)=b_{c}\left(c_{1}(t)\right)-m L
$$

for all $m \in \mathbb{Z}$. Since $b_{c}$ is 1-Lipschitz, the second assertion follows. q.e.d. 


\section{Busemann functions of axes of isometries}

The goal of this section in to prove the following.

Proposition 3.1. Let $\gamma \in \Gamma \backslash\{e\}$, and let $c$ and $c_{1}$ be axes of $\gamma$. Then $b_{c}-b_{c_{1}}$ is constant on $M$.

This proposition was proved in the analytic case in $[\mathbf{8}$, Proposition $2.5]$. The analyticity assumption is used in [8] to ensure some regularity of the set $A_{\gamma}$, namely it implies that this set is locally rectifiably path connected (see $[\mathbf{8}]$ for the definition). This property and local analysis of Busemann functions on $A_{\gamma}$ yields the result. We do not know if $A_{\gamma}$ is locally rectifiably path connected if the metric is only $C^{\infty}$. We work around this issue by more delicate analysis of the behavior of Busemann functions in a neighborhood of $A_{\gamma}$ (Lemma 3.2).

For a geodesic $c$ in $M$, denote by $b_{c}^{-}$the Busemann function of the reverse geodesic $t \mapsto c(-t)$ and let $b_{c}^{0}=b_{c}+b_{c}^{-}$. The definition of a Busemann function and the triangle inequality imply that $b_{c}^{0}(x) \geq 0$ for all $x \in M$.

Lemma 3.2. Let $c$ be an axis of $\gamma$ and $L=\min d_{\gamma}$. Define $f(x)=$ $d_{\gamma}(x)-L$ for $x \in M$. Then there exists $C>0$ such that

$$
\left|b_{c}^{0}(x)-b_{c}^{0}(y)\right| \leq C\left(d(x, y)^{2}+f(x)+f(y)\right)
$$

for all $x, y \in M$.

Proof. Note that $\left|b_{c}^{0}(x)-b_{c}^{0}(y)\right| \leq 2 d(x, y)$ since Busemann functions are 1-Lipschitz. Therefore we may assume that $d(x, y)$ is small; more precisely, $d(x, y) \leq L / 2$. Indeed, if $d(x, y)>L / 2$ then $\left|b_{c}^{0}(x)-b_{c}^{0}(y)\right| \leq$ $2 d(x, y) \leq 4 L^{-1} d(x, y)^{2}$, so (3.1) is satisfied for any $C \geq 4 L^{-1}$. We may also assume that $f(x) \leq 1$ and $f(y) \leq 1$, otherwise

$$
\left|b_{c}^{0}(x)-b_{c}^{0}(y)\right| \leq 2 d(x, y) \leq L \leq L(f(x)+f(y)),
$$

so (3.1) is satisfied for any $C \geq L$.

Let $x_{+}=\gamma x$ and $x_{-}=\gamma^{-1} x$. Then

$$
d\left(x, x_{+}\right)=d\left(x, x_{-}\right)=d_{\gamma}(x)=L+f(x) .
$$

By Lemma 2.4(1), we have $b_{c}(x)=b_{c}\left(x_{+}\right)+L$ and $b_{c}^{-}(x)=b_{c}^{-}\left(x_{-}\right)+L$. Since Busemann functions are 1-Lipschitz, it follows that

$$
b_{c}(y)-b_{c}(x)=b_{c}(y)-b_{c}\left(x_{+}\right)-L \leq d\left(y, x_{+}\right)-L
$$

and

$$
b_{c}^{-}(y)-b_{c}^{-}(x)=b_{c}(y)-b_{c}^{-}\left(x_{-}\right)-L \leq d\left(y, x_{-}\right)-L .
$$

Summing these two inequalities yields

$$
b_{c}^{0}(y)-b_{c}^{0}(x) \leq d\left(y, x_{+}\right)+d\left(y, x_{-}\right)-2 L .
$$

Our plan is to estimate the right-hand side of this inequality. 
For $p, q \in M$, denote by $\overrightarrow{p q}$ the initial velocity vector of the unique geodesic connecting $p$ to $q$. That is, $\overrightarrow{p q}$ is the unit vector in $T_{p} M$ positively proportional to $\exp _{p}^{-1}(q)$. Let $v_{+}=\overrightarrow{x x_{+}}, v_{-}=\overrightarrow{x x_{-}}$and $z \in M$ be any point such that $d(x, z) \leq L / 2$. Then, by the first variation formula and the second order Taylor formula,

$$
\begin{aligned}
d\left(z, x_{ \pm}\right) & \leq d\left(x, x_{ \pm}\right)-\left\langle\overrightarrow{x z}, v_{ \pm}\right\rangle \cdot d(x, z)+C_{1} d(x, z)^{2} \\
& =L+f(x)-\left\langle\overrightarrow{x z}, v_{ \pm}\right\rangle \cdot d(x, z)+C_{1} d(x, z)^{2}
\end{aligned}
$$

and therefore

$d\left(z, x_{+}\right)+d\left(z, x_{-}\right) \leq 2 L+2 f(x)-\left\langle\overrightarrow{x z}, v_{+}+v_{-}\right\rangle \cdot d(x, z)+2 C_{1} d(x, z)^{2}$, where $C_{1}>0$ is a constant depending only on $M$ and $L$. (This constant is just an upper bound for the second derivative of the distance to $x_{ \pm}$ on the geodesic segment $[x, z]$. It is uniform in $x$ because $M$ admits a co-compact action by isometries and $L \leq d\left(x, x_{ \pm}\right) \leq L+1$.)

For $z=y,(3.2)$ and (3.3) imply that

$$
\begin{aligned}
b_{c}^{0}(y)-b_{c}^{0}(x) & \leq 2 f(x)-\left\langle\overrightarrow{x y}, v_{+}+v_{-}\right\rangle \cdot d(x, y)+2 C_{1} d(x, y)^{2} \\
& \leq 2 f(x)+\sigma \cdot d(x, y)+2 C_{1} d(x, y)^{2}
\end{aligned}
$$

where $\sigma=\left|v_{+}+v_{-}\right|$.

It remains to estimate $\sigma$. Consider a point $z=\exp _{x}\left(t_{0} v\right)$, where $v \in T_{x} M$ is the unit vector positively proportional to $v_{+}+v_{-}$(or any unit vector if $\left.v_{+}+v_{-}=0\right)$ and $t_{0}=\sqrt{f(x) / C_{1}}$. We may assume that $C_{1}$ is sufficiently large so that $t_{0} \leq L / 2$ (recall that $f(x) \leq 1$ ). For the so-defined $z,(3.3)$ takes the form

$$
d\left(z, x_{+}\right)+d\left(z, x_{-}\right) \leq 2 L+2 f(x)-\sigma t_{0}+2 C_{1} t_{0}^{2} .
$$

On the other hand,

$$
d\left(z, x_{+}\right)+d\left(z, x_{-}\right) \geq d\left(x_{+}, x_{-}\right)=d_{\gamma^{2}}\left(x_{-}\right) \geq \min d_{\gamma^{2}}=2 L
$$

by the triangle inequality and Lemma 2.1(3) (recall that $x_{+}=\gamma^{2} x_{-}$). Hence

$$
0 \leq 2 f(x)-\sigma t_{0}+2 C_{1} t_{0}^{2}=-\sigma \sqrt{f(x) / C_{1}}+4 f(x),
$$

or, equivalently, $\sigma \leq 4 \sqrt{C_{1} f(x)}$. Therefore

$$
\sigma \cdot d(x, y) \leq 4 \sqrt{C_{1} f(x) d(x, y)^{2}} \leq 2 f(x)+2 C_{1} d(x, y)^{2} .
$$

Plugging this into (3.4) yields

$$
b_{c}^{0}(y)-b_{c}^{0}(x) \leq 4 f(x)+4 C_{1} d(x, y)^{2}
$$

and, by switching the roles of $x$ and $y$,

$$
b_{c}^{0}(x)-b_{c}^{0}(y) \leq 4 f(y)+4 C_{1} d(x, y)^{2} .
$$

Therefore (3.1) is satisfied for $C \geq \max \left\{4 C_{1}, 4\right\}$.

q.e.d. 
The following lemma is purely analytic and local. It does not use the assumption that $M$ is co-compact and free of conjugate points.

Lemma 3.3. Let $M$ be any Riemannian manifold, $f \in C^{\infty}(M)$ and $g: M \rightarrow \mathbb{R}$ a continuous function such that

$$
|g(x)-g(y)| \leq C\left(d(x, y)^{2}+|f(x)|+|f(y)|\right)
$$

for some constant $C>0$ and all $x, y \in M$. Let $X=f^{-1}(0)$. Then the set $g(X) \subset \mathbb{R}$ has zero Lebesgue measure.

Proof. We argue by induction in $n=\operatorname{dim} M$. The case $n=0$ is trivial. Assume that $n \geq 1$ and the lemma holds for all $(n-1)$-dimensional manifolds.

Since $M$ can be covered by countably many coordinate neighborhoods, we may assume that $M$ is an open ball in $\mathbb{R}^{n}$ and $f$ extends to a smooth function on a compact set. Let $Z$ be the set of points in $X$ where all derivatives of $f$ vanish, and let $Y=X \backslash Z$.

The set $Y$ is contained in a countable union of $(n-1)$-dimensional smooth submanifolds. Indeed, for each multi-index $I=\left(i_{1}, \ldots, i_{k}\right) \in$ $\{1, \ldots, n\}^{k}, k \geq 0$, consider the partial derivative $f_{I}=\frac{\partial^{k} f}{\partial x_{1} \ldots \partial x_{k}}$. Let $\Sigma_{I}$ be the set of points in $M$ where the function $f_{I}$ vanishes but its first derivative does not. Since $f_{I} \in C^{\infty}, \Sigma_{I}$ is a smooth $(n-1)$-dimensional submanifold, and $Y$ is contained in the union of all such submanifolds.

Applying the induction hypothesis to $\Sigma_{I}$ in place of $M$ yields that $g\left(X \cap \Sigma_{I}\right)$ is a set of measure zero for every multi-index $I$. Therefore $g(Y)$ has measure zero.

It remains to handle the set $g(Z)$. Since $f$ and all its derivatives up to the order $2 n$ vanish on $Z$ and are bounded on $M$, there is a constant $C_{1}>0$ such that

$$
|f(x)|=|f(x)-f(z)| \leq C_{1}|x-z|^{2 n}
$$

for all $x \in M$ and $z \in Z$. We are going to show that

$$
\left|g(z)-g\left(z^{\prime}\right)\right| \leq C_{2}\left|z-z^{\prime}\right|^{n+1}
$$

for some constant $C_{2}>0$ and all $z, z^{\prime} \in Z$ such that $\left|z-z^{\prime}\right| \leq 1$. Let $\delta=\left|z-z^{\prime}\right|$ and pick a positive integer $N$ such that $\delta^{1-n} \leq N \leq 2 \delta^{1-n}$. Divide the segment $\left[z, z^{\prime}\right]$ into $N$ segments $\left[x_{i-1}, x_{i}\right], i=1, \ldots, N$, of equal lengths. Note that

$$
\left|x_{i}-x_{i-1}\right|=\delta / N \leq \delta^{n}
$$

by the choice of $N$, and

$$
\left|f\left(x_{i}\right)\right|+\left|f\left(x_{i-1}\right)\right| \leq C_{1}\left|x_{i}-z\right|^{2 n}+C_{1}\left|x_{i-1}-z\right|^{2 n} \leq 2 C_{1} \delta^{2 n}
$$

by (3.6). Substituting $x_{i}$ and $x_{i-1}$ for $x$ and $y$ in (3.5) yields that $\left|g\left(x_{i}\right)-g\left(x_{i-1}\right)\right| \leq C\left(\left|x_{i}-x_{i-1}\right|^{2}+\left|f\left(x_{i}\right)\right|+\left|f\left(x_{i-1}\right)\right|\right) \leq C\left(1+2 C_{1}\right) \delta^{2 n}$. 
Hence

$\left|g(z)-g\left(z^{\prime}\right)\right|=\left|\sum g\left(x_{i}\right)-g\left(x_{i-1}\right)\right| \leq C\left(1+2 C_{1}\right) \delta^{2 n} N \leq 2 C\left(1+2 C_{1}\right) \delta^{n+1}$.

Thus (3.7) holds for $C_{2}=2 C\left(1+2 C_{1}\right)$.

The inequality (3.7) implies that

$$
\operatorname{dim}_{H}(g(Z)) \leq \frac{\operatorname{dim}_{H}(Z)}{n+1} \leq \frac{\operatorname{dim}_{H}(M)}{n+1}=\frac{n}{n+1}<1
$$

where $\operatorname{dim}_{H}$ denotes the Hausdorff dimension. Hence the one-dimensional measure of $g(Z)$ is zero and the lemma follows.

q.e.d.

Corollary 3.4. Let $c$ be an axis of $\gamma$. Then $b_{c}^{0}=0$ on $A_{\gamma}$.

Proof. Let $L=\min d_{\gamma}$. Then Lemma 3.2 asserts that the functions $f=d_{\gamma}-L$ and $g=b_{c}^{0}$ satisfy the assumptions of Lemma 3.3. Since $A_{\gamma}=f^{-1}(0)$, Lemma 3.3 implies that $b_{c}^{0}\left(A_{\gamma}\right)$ is a set of measure zero in $\mathbb{R}$. By Lemma 2.2, $A_{\gamma}$ and hence $b_{c}^{0}\left(A_{\gamma}\right)$ are connected. Since the set $b_{c}^{0}\left(A_{\gamma}\right)$ is connected and has measure zero, it is a single point on the real line. This means that $b_{c}^{0}$ is constant on $A_{\gamma}$. Since $c(0) \in A_{\gamma}$ and $b_{c}^{0}(c(0))=0$, this constant is zero. $\quad$ q.e.d.

Proof of Proposition 3.1. Let $c$ and $c_{1}$ be axes of $\gamma$. Changing the origin of $c_{1}$ we may assume that $b_{c}\left(c_{1}(0)\right)=0$. Then we have to prove that $b_{c}=b_{c_{1}}$.

First we show that the relation $b_{c}\left(c_{1}(0)\right)=0$ is symmetric; i.e., it implies that $b_{c_{1}}(c(0))=0$. Since $c_{1}(0) \in A_{\gamma}$, we have $b_{c}^{0}\left(c_{1}(0)\right)=0$ by Corollary 3.4; hence $b_{c}^{-}\left(c_{1}(0)\right)=0$. This means that

$$
d\left(c_{1}(0), c(-t)\right)=t+\varepsilon(t)
$$

where $\varepsilon(t) \rightarrow 0$ as $t \rightarrow+\infty$. Since $b_{c_{1}}(c(-t)) \leq d\left(c(-t), c_{1}(0)\right)$, it follows that $b_{c_{1}}(c(-t)) \leq t+\varepsilon(t)$. This and Lemma 2.4(2) imply that

$$
b_{c_{1}}(c(0))=b_{c_{1}}(c(-t))-t \leq \varepsilon(t)
$$

for all $t>0$. Therefore $b_{c_{1}}(c(0)) \leq 0$. Similarly, $b_{c_{1}}^{-}(c(0)) \leq 0$ (recall that $b_{c}^{-}\left(c_{1}(0)\right)=0$ so the argument applies to the reverse geodesics as well). Both inequalities must turn to equalities because $b_{c_{1}}+b_{c_{1}}^{-} \geq 0$. Thus $b_{c_{1}}(c(0))=0$ as claimed.

Let $x \in M$ and $\varepsilon>0$. By the definition of Busemann function, there exists $t_{1} \in \mathbb{R}$ such that

$$
d\left(x, c_{1}\left(t_{1}\right)\right)<b_{c_{1}}(x)+t_{1}+\varepsilon .
$$

Since $b_{c}\left(c_{1}(0)\right)=0$, Lemma $2.4(2)$ implies that $b_{c}\left(c_{1}\left(t_{1}\right)\right)=-t_{1}$; hence

$$
d\left(c_{1}\left(t_{1}\right), c\left(t_{0}\right)\right)<t_{0}-t_{1}+\varepsilon
$$

for a sufficiently large $t_{0} \in \mathbb{R}$. Therefore

$$
d\left(x, c\left(t_{0}\right)\right) \leq d\left(x, c_{1}\left(t_{1}\right)\right)+d\left(c_{1}\left(t_{1}\right), c\left(t_{0}\right)\right)<b_{c_{1}}(x)+t_{0}+2 \varepsilon
$$

and hence $b_{c}(x) \leq b_{c_{1}}(x)$ since $\varepsilon$ is arbitrary. 
Swapping $c$ and $c_{1}$ in this argument yields that $b_{c_{1}}(x) \leq b_{c}(x)$. Thus $b_{c}=b_{c_{1}}$ and the proposition follows.

q.e.d.

\section{Virtual splitting of centralizer}

Lemma 4.1. Let $\alpha \in Z(\gamma)$ and $c$ be an axis of $\gamma$. Then $b_{c}(\alpha x)-b_{c}(x)$ does not depend on $x \in M$.

Proof. This is [8, Corollary 2.6] without the analyticity assumption. Observe that $\alpha^{-1} c$ is an axis of $\gamma$, indeed, if $L=\min d_{\gamma}$ then

$$
\gamma \alpha^{-1} c(t)=\alpha^{-1} \gamma c(t)=\alpha^{-1} c(t+L) \text {. }
$$

Therefore $b_{\alpha^{-1} c}-b_{c}$ is constant by Proposition 3.1. Since $b_{\alpha^{-1} c}(x)=$ $b_{c}(\alpha x)$, the lemma follows. q.e.d.

Corollary 4.2. There exists a homomorphism $h: Z(\gamma) \rightarrow \mathbb{R}$ such that $h(\gamma) \neq 0$.

Proof. Let $c$ be an axis of $\gamma$. For $\alpha \in Z(\gamma)$, define

$$
h(\alpha)=b_{c}(\alpha x)-b_{c}(x),
$$

where $x \in M$ is an arbitrary point. By Lemma $4.1, h(\alpha)$ is well defined. For $\alpha, \beta \in Z(\gamma)$ we have

$h(\alpha \beta)=b_{c}(\alpha \beta x)-b_{c}(x)=b_{c}(\alpha \beta x)-b_{c}(\beta x)+b_{c}(\beta x)-b_{c}(x)=h(\alpha)+h(\beta)$.

Thus $h$ is a homomorphism. By Lemma 2.4(1), $h(\gamma)=-\min d_{\gamma} \neq 0$. q.e.d.

Recall that $H_{1}(Z(\gamma))=Z(\gamma) /[Z(\gamma), Z(\gamma)]$. We denote by $\pi$ the projection from $Z(\gamma)$ to $H_{1}(Z(\gamma))$.

Corollary 4.3. $\pi(\gamma)$ is non-torsion (i.e., has infinite order) in $H_{1}(Z(\gamma))$.

Proof. Since $\mathbb{R}$ is commutative, the homomorphism $h$ from Corollary 4.2 factors as $h=\bar{h} \circ \pi$, where $\bar{h}$ is a homomorphism from $H_{1}(Z(\gamma))$ to $\mathbb{R}$. Since $\bar{h}(\pi(\gamma)) \neq 0, \pi(\gamma)$ has infinite order.

q.e.d.

Proof of Theorem A. Recall that by Remark 2.3, $Z(\gamma)$ is finitely generated and hence so is $H_{1}(Z(\gamma))$. By Corollary 4.3 and the classification of finitely generated abelian groups, $\pi(\gamma)$ belongs to a finite index subgroup $H<H_{1}(Z(\gamma))$ that is isomorphic to $\mathbb{Z}^{k}$ so that $\pi(\gamma)$ is mapped to $(1,0, \ldots, 0) \in \mathbb{Z}^{k}$ by this isomorphism. Let $p: H \cong \mathbb{Z}^{k} \rightarrow \mathbb{Z}$ be first coordinate projection. Then $G:=\pi^{-1}(H)$ is a finite index subgroup of $Z(\gamma)$ and $\phi=p \circ \pi: G \rightarrow \mathbb{Z}$ is a homomorphism sending $\gamma$ to $1 \in \mathbb{Z}$. The existence of such a homomorphism and the fact that $\gamma$ belongs to the center of $G$ imply that $G \cong \mathbb{Z} \times G^{\prime}$, where the $\mathbb{Z}$ factor corresponds to the subgroup generated by $\gamma$ and $G^{\prime}=\operatorname{ker} \phi$.

q.e.d. 


\section{Examples}

In this section we will give some new examples of manifolds that can be shown not to admit metrics without conjugate points using Theorem A.

Example 5.1. Let $S_{g}^{2}$ be a closed orientable surface of genus $g>1$ equipped with any Riemannian metric. Let $\bar{M}^{3}=T^{1} S_{g}^{2}$ be the unit tangent bundle to $S_{g}^{2}$. Then $\bar{M}^{3}$ does not admit a metric without conjugate points.

Indeed, since the Euler class of $S_{g}^{2}$ is not equal to zero, $\pi_{1}(\bar{M})$ is a nontrivial central extension $1 \rightarrow \mathbb{Z} \rightarrow \pi_{1}(\bar{M}) \rightarrow \pi_{1}\left(S_{g}^{2}\right) \rightarrow 1$. A nontrivial central element $\gamma \in \pi_{1}(\bar{M})$ satisfies $Z(\gamma)=\pi_{1}(\bar{M})$, but there is no virtual splitting of $\pi_{1}(\bar{M})$ over $\gamma$. By Theorem A it follows that $\bar{M}$ does not admit a metric without conjugate points. On the other hand, $\pi_{1}(\bar{M})$ is semi-hyperbolic [1] and therefore it satisfies the previously known restrictions on fundamental groups of manifolds without conjugate points proved in [8], namely, every abelian subgroup of $\pi_{1}(\bar{M})$ is straight and every solvable subgroup of $\pi_{1}(\bar{M})$ is virtually abelian.

Recall that the key step in the proof of Theorem A is Proposition 3.1. It was proved in $[8]$ under the assumption that $A_{\gamma}$ is locally rectifiably path connected. As was discussed earlier this can be guaranteed if the metric is real analytic. Another condition that obviously ensures it is if $\gamma$ belongs to the center of $\pi_{1}(\bar{M})$. Indeed, if $\gamma \in Z\left(\pi_{1}(\bar{M})\right)$ then $d_{\gamma}$ descends to a well-defined function on $M$ and hence it is bounded above and attains its maximum. By Lemma 2.1(4), this means that max $d_{\gamma}=$ $\min d_{\gamma}$; i.e., $d_{\gamma}$ is constant. That means that $A_{\gamma}=M$, which is obviously rectifiably path connected. Therefore one can prove that $\bar{M}$ admits no metric without conjugate points without using Proposition 3.1.

Next we will give an example that has no finite index subgroups with nontrivial center and requires the full strength of Proposition 3.1 and Theorem A.

Example 5.2. Let $S_{g}^{2}$ be a closed orientable surface of genus $g>1$, $\bar{M}^{3}=T^{1} S_{g}$ and $F=S_{g} \# S_{g}$. Our example is a 5 -manifold $N$ fibering over $\bar{M}$ with fiber $F$. We need some preliminaries before we carry out the construction.

First let us recall some basic facts about diffeomorphism groups of surfaces. The mapping class group $\operatorname{Mod}(F)$ is defined as $\pi_{0}(\operatorname{Diff}(F))$. It can be alternatively described as $\operatorname{Diff}(F) / \operatorname{Diff}_{0}(F)$ ) where $\operatorname{Diff}_{0}(F)$ is the identity component of $\operatorname{Diff}(F)$. By a classical result of Dehn and Nielsen, $\operatorname{Mod}(F)$ is isomorphic to the group

$$
\operatorname{Out}\left(\pi_{1}(F)\right)=\operatorname{Aut}\left(\pi_{1}(F)\right) / \operatorname{Inn}\left(\pi_{1}(F)\right)
$$


of outer automorphisms of $\pi_{1}(F)$. For a once-punctured surface $F \backslash\{p t\}$, we have $\operatorname{Mod}(F \backslash\{p t\}) \cong \operatorname{Aut}\left(\pi_{1}(F)\right)$.

It is well known that $\operatorname{Diff}_{0}(F)$ is contractible $[\mathbf{9}]$.

Lemma 5.3. There exists a monomorphism $\rho: \pi_{1}(\bar{M}) \rightarrow \operatorname{Mod}(F)$ that admits a lift to a monomorphism $\bar{\rho}: \pi_{1}(\bar{M}) \rightarrow \operatorname{Mod}(F \backslash\{p t\})$.

Proof. Pick $p \in S_{g}$ and $v \in T_{p}^{1} S_{g}$. Let $\operatorname{Diff}\left(S_{g}, v\right)$ be the subgroup of $\operatorname{Diff}\left(S_{g}\right)$ fixing $v$. Consider the fibration $\operatorname{Diff}\left(S_{g}, v\right) \rightarrow \operatorname{Diff}\left(S_{g}\right) \stackrel{e v}{\rightarrow} \bar{M}^{3}$, where the last map is just the evaluation map on $v$. Look at the long exact homotopy sequence of this fibration

$$
\ldots \rightarrow \pi_{1}\left(\operatorname{Diff}\left(S_{g}\right)\right) \rightarrow \pi_{1}\left(\bar{M}^{3}\right) \stackrel{i}{\rightarrow} \pi_{0}\left(\operatorname{Diff}\left(S_{g}, v\right)\right) \rightarrow \pi_{0}\left(\operatorname{Diff}\left(S_{g}\right)\right)
$$

As mentioned earlier, $\operatorname{Diff}_{0}\left(S_{g}\right)$ is contractible; therefore $\pi_{1}\left(\operatorname{Diff}\left(S_{g}\right)\right)$ $=1$ and hence $\pi_{1}\left(\bar{M}^{3}\right)$ injects as a subgroup into $\pi_{0}\left(\operatorname{Diff}\left(S_{g}, v\right)\right)$. Next choose an $\varepsilon>0$ much smaller than the injectivity radius of $S_{g}$ and let $S^{+}=S_{g} \backslash B_{\varepsilon}(p)$. Then $\operatorname{Diff}\left(S_{g}, v\right)$ is naturally isomorphic to $\operatorname{Diff}\left(S^{+}, \partial S^{+}\right)$, the group of diffeomorphisms of $S^{+}$fixing the boundary $\partial S^{+}$pointwise. Let $F=S^{+} \cup_{\partial S^{+}} S^{-}$, where $S^{-}$is another copy of $S^{+}$(i.e., $F$ is the double of $S^{+}$along its boundary). Then $\operatorname{Diff}\left(S^{+}, \partial S^{+}\right)$ naturally embeds into $\operatorname{Diff}(F)$ by extending the diffeomorphisms to $S^{-}$ by identity. This induces a map $j: \pi_{0}\left(\operatorname{Diff}\left(S^{+}, \partial S^{+}\right)\right) \rightarrow \pi_{0}(\operatorname{Diff}(F))$ that is also injective. Then $\rho=j \circ i: \pi_{1}(\bar{M}) \rightarrow \operatorname{Mod}(F)$ is injective.

The above construction (originally due to Mess [16]) is borrowed from [13, Section 4.1]; see also [2].

Next observe that if instead of gluing $S^{-}$to $S^{+}$we glue $S^{-} \backslash\{p t\}$, the same argument gives an embedding $\pi_{1}(\bar{M}) \rightarrow \operatorname{Mod}(F \backslash\{p t\})$ so that the above map $\rho: \pi_{1}(\bar{M}) \rightarrow \operatorname{Out}\left(\pi_{1}(F)\right)$ lifts to a map $\bar{\rho}: \pi_{1}(\bar{M}) \rightarrow$ $\operatorname{Mod}(F \backslash\{p t\})$ and both of these are injections.

q.e.d.

Let $B \operatorname{Diff}(F)=E / \operatorname{Diff}(F)$ be the classifying space of $\operatorname{Diff}(F)$ (here $E$ is contractible). Then the projection map $p: E \rightarrow B \operatorname{Diff}(F)$ factors through the map

$$
E \rightarrow E / \operatorname{Diff}_{0}(F) \stackrel{\bar{p}}{\rightarrow} E / \operatorname{Diff}(F)=B \operatorname{Diff}(F),
$$

where $\bar{p}$ is just the quotient by $\left.\operatorname{Diff}(F) / \operatorname{Diff}_{0}(F)\right)=\operatorname{Mod}(F)$. Since $\operatorname{Diff}_{0}(F)$ is contractible then so is $E / \operatorname{Diff}_{0}(F)$ and therefore $B \operatorname{Diff}(F)$ can be identified with $B \operatorname{Mod}(F)$, which we will do from now on. Therefore, for any $\mathrm{CW}$ complex $B$ any map $f: B \rightarrow B \operatorname{Mod}(F)$ (which up to homotopy is determined by the map on the fundamental groups) gives rise to a pullback bundle $\operatorname{Diff}(F) \rightarrow X \rightarrow B$ and its associated bundle $F \rightarrow N \rightarrow B$ coming from the natural action of $\operatorname{Diff}(F)$ on $F$.

Now consider the map $\bar{M} \rightarrow B \operatorname{Mod}(F)$ corresponding to the monomorphism $\rho: \pi_{1}(\bar{M}) \rightarrow \operatorname{Mod}(F)$ from Lemma 5.3 and pull back the universal $F$ bundle. This gives us a 5-manifold $N$ fibering over $\bar{M}$ with 
fiber $F$. Clearly $N$ is aspherical and its fundamental groups fits into a short exact sequence

$$
1 \rightarrow \pi_{1}(F) \rightarrow \pi_{1}(N) \rightarrow \pi_{1}(\bar{M}) \rightarrow 1 .
$$

Since $\rho$ can be lifted to $\bar{\rho}: \pi_{1}(\bar{M}) \rightarrow \operatorname{Mod}(F \backslash\{p t\}) \cong \operatorname{Aut}\left(\pi_{1}(F)\right)$, this exact sequence splits, i.e., $\pi_{1}(N)$ is isomorphic to the semidirect product $\pi_{1}(F) \rtimes_{\bar{\rho}} \pi_{1}(\bar{M})$.

Since $\bar{\rho}$ is injective, $\pi_{1}(N)$ has trivial center. On the other hand, it contains $\pi_{1}(\bar{M})$ as a subgroup and therefore it has an element whose centralizer does not virtually split. Therefore, $N$ does not admit a metric without conjugate points by Theorem A. Lastly note that since $\rho$ is injective, the whole group $\pi_{1}(N)$ injects into $\operatorname{Aut}\left(\pi_{1}(F)\right)$ via the conjugation action. Also recall that $\operatorname{Aut}\left(\pi_{1}(F)\right) \cong \operatorname{Mod}(F \backslash\{p t\})$. It is well known that mapping class groups of hyperbolizable surfaces satisfy the property that all their abelian subgroups are straight (see $[\mathbf{1 0}]$ and $[\mathbf{1 2}$, Lemma 8.7]) and all their solvable subgroups are virtually abelian [3]. Of course the same is therefore true for all their subgroups and hence it is true for $\pi_{1}(N)$.

\section{Solvability of word and conjugacy problems}

It is very well known that fundamental groups of manifolds of nonpositive curvature have solvable word and conjugacy problems. We observe that the same is true for fundamental groups of closed manifolds without conjugate points.

Theorem 6.1. Let $\bar{M}$ be a closed manifold with a Riemannian metric without conjugate points. Then $\pi_{1}(\bar{M})$ has solvable word and conjugacy problems.

Proof. The proof is a straightforward combination of known results. The key is the following Lemma.

Lemma 6.2. Let $c_{0}, c_{1}: S^{1} \rightarrow \bar{M}$ be two freely homotopic rectifiable loops with length $L\left(c_{i}\right)<C$ for $i=0,1$. Then there exists a free homotopy $F: S^{1} \times[0,1] \rightarrow \bar{M}$ from $c_{0}$ to $c_{1}$ through loops $c_{t}$ such that $L\left(c_{t}\right)<C$ for any $t \in[0,1]$.

Proof. Lifting the homotopy to the universal cover $M$ we get a homotopy between two curves $\tilde{c}_{0}, \tilde{c}_{1}:[0,1] \rightarrow M$. Since the metric has no conjugate points, it is easy to connect each $\tilde{c}_{i}, i=1,2$, to the (unique) geodesic segment $\left[\tilde{c}_{i}(0), \tilde{c}_{i}(1)\right]$ by a homotopy fixing the endpoints and not increasing lengths. (For example, consider a family of curves $\left\{\tilde{c}_{i, t}\right\}, t \in[0,1]$, where $\tilde{c}_{i, t}$ is the concatenation of the geodesic segment $\left[\tilde{c}_{i}(0), \tilde{c}_{i}(t)\right]$ and the $\left.\operatorname{arc} \tilde{c}_{i}\right|_{[t, 1]}$ of $\tilde{c}_{i}$.) Thus we may assume that $\tilde{c}_{0}$ and $\tilde{c}_{1}$ are geodesics.

Let $\gamma$ be the element of the deck transformation group such that $\gamma\left(\tilde{c}_{0}(0)\right)=\tilde{c}_{0}(1)$; then $\gamma\left(\tilde{c}_{1}(0)\right)=\tilde{c}_{1}(1)$. Thus, by our assumption, both 
$\tilde{c}_{0}(0)$ and $\tilde{c}_{1}(0)$ belong to the set $\left\{d_{\gamma}<C\right\}$. By Lemma 2.1(4) and the proof of Lemma 2.2, the set $\left\{d_{\gamma}<C\right\}$ is path connected and therefore we can find a path $\alpha:[0,1] \rightarrow\left\{d_{\gamma}<C\right\}$ such that $\alpha(0)=\tilde{c}_{0}(0)$ and $\alpha(1)=\tilde{c}_{1}(0)$. Connect $\alpha(s)$ to $\gamma(\alpha(s))$ by shortest geodesics for all $s \in$ $[0,1]$. They are unique and vary continuously since $M$ has no conjugate points. Projecting this family of geodesics to $\bar{M}$ produces a homotopy with the desired properties.

q.e.d.

We are indebted to Ilya Kapovich for providing the following argument that the property provided by Lemma 6.2 implies solvability of the word problem in $\pi_{1}(\bar{M})$. Since the argument is apparently well-known we only give a brief outline.

It is well-known that to prove solvability of the word problem in a finitely presented group, it is enough to show that its Dehn function has a recursive upper bound (see for example [4, Theorem 1.5.8, p. 104]).

We claim that Lemma 6.2 yields an exponential upper bound on the Dehn function of $\pi_{1}(\bar{M})$.

Fix a finite presentation $\langle F \mid R\rangle$ of $\Gamma=\pi_{1}(\bar{M}, p)$ and a $K$-quasiisometry between $M$ and the Caley graph of $\Gamma$ with respect to that presentation sending $p$ to $e$.

Let $F: S^{1} \times[0,1] \rightarrow \bar{M}$ be a homotopy between a constant loop $c_{0}$ at $p$ and a loop $c_{1}$. Let $C=2 L\left(c_{1}\right)$. Since we are only interested in large scale estimates we can assume that $C \geq 2 \operatorname{diam} \bar{M}$. By Lemma 6.2 we can change $F$ to a free homotopy such that $L\left(c_{t}\right) \leq C$ for any $t$.

Consider a sufficiently fine subdivision of $[0,1]$ given by $0=t_{0}<$ $t_{1}<\ldots<t_{N}=1$. For every $j=0, \ldots N$, connect $c_{t_{j}}(0)$ to the fixed base point $p=c_{0}(0)$ by a shortest geodesic. For every $j$ this produces a nullhomotopic loop $c_{j}^{\prime}$ based at $p$ of length $\leq C+2 \operatorname{diam}(\bar{M})$. Look at the corresponding curves in the Caley graph and the words they represent in the presentation $\langle F \mid R\rangle$. They all have lengths $\leq K(C+2 \operatorname{diam}(\bar{M})) \leq$ $2 K C$. The ball of radius $R$ in the Caley graph has at most $\exp \left(C_{2} \cdot R\right)$ vertices for some explicit constant $C_{2}$ depending on $|F|$. Therefore the loops $c_{j}^{\prime}$ produce at most $\exp \left(2 C_{2} K C\right)$ distinct words in the group. Therefore, if the original homotopy was "too long" we can cut out the parts between repeating words and reduce its length. This produces a homotopy in the Caley graph of area $\leq \exp \left(C_{3} \cdot C\right)$ for some easily computable constant $C_{3}=C_{3}\left(C_{2}, K,|F|+|R|\right)$. Therefore the Dehn function grows at most exponentially.

Lastly, by [5, section 1.11, p. 446], if $\pi_{1}(\bar{M})$ has solvable word problem and satisfies the conclusion of Lemma 6.2, then it also has solvable conjugacy problem.

q.e.d.

REMARK 6.3. One can also prove the solvability of the word problem in $\Gamma$ as follows. Since $\bar{M}$ is compact we have that $|\sec (M)| \leq K$ for some $K>0$, which trivially implies that for any $p \in M$ the exponential 
map $\exp _{p}: T_{p} M \rightarrow M$ is $\exp \left(C_{1} R\right)$ Lipschitz on the $R$-ball $B_{R}(0) \subset$ $T_{p} M$ for some $C_{1}>0$ and any $R>0$. Using that geodesics in $M$ are globally distance minimizing, it is not hard to show that $\exp _{p}^{-1}$ is also $\exp \left(C_{2} R\right)$ Lipschitz, which implies an exponential bound on the isoperimetric function in $M$ and hence the Dehn function of $\Gamma$ also grows at most exponentially.

It is well-known that Dehn functions of $C A T(0)$-groups grow at most quadratically. It is reasonable to expect that the same should be true for fundamental groups of closed manifolds without conjugate points but we have been unable to prove it.

\section{3-manifolds without focal points}

Recall that a Riemannian manifold $M$ is said to have no focal points if every embedded shortest geodesic $c:(a, b) \rightarrow M$ has no focal points when viewed as a submanifold of $M$. The class of manifolds without focal points is contained in the class of manifolds without conjugate points and contains all nonpositively curved manifolds. It is therefore natural to wonder if closed manifolds without focal points always admit nonpositively curved metrics.

The main result of this section is the following theorem, which answers this question affirmatively in dimension 3 .

Theorem 7.1. Let $M$ be a closed 3-manifold. Then $M$ admits a Riemannian metric without focal points if and only if it admits a Riemannian metric of nonpositive sectional curvature.

One of the main technical tools in the proof is the following result due to O'Sullivan [17].

Theorem 7.2 (Flat torus theorem). [17, Theorem 2] Let $M$ be a closed Riemannian manifold without focal points. Let $A \leq \pi_{1}(M)$ be a solvable subgroup. Then there exists a flat totally geodesically embedded space form $i: F^{k} \hookrightarrow M$ such that $i_{*}: \pi_{1}\left(F^{k}\right) \rightarrow \pi_{1}(M)$ is injective and $A$ is a finite index subgroup in $i_{*}\left(\pi_{1}\left(F^{k}\right)\right)$.

We also need the following result from the same paper.

Proposition 7.3. [17, Proposition 5] Let $\bar{M}$ be a closed manifold without focal points and $\gamma$ a nontrivial element of the deck transformation group $\Gamma \cong \pi_{1}(\bar{M})$. Then

1) The union of axes $A_{\gamma}$ is convex and closed in the universal cover $M$.

2) $A_{\gamma}$ is isometric to $\mathbb{R} \times N$, where $N$ is a smooth submanifold of $\bar{M}$, possibly with boundary. The action of $\gamma$ preserves this splitting and acts by translations by $\min d_{\gamma}$ on the $\mathbb{R}$-factors.

Proof of Theorem 7.1. To simplify the exposition we will only give the proof for orientable manifolds. The non-orientable case is treated similarly. Alternatively we can appeal to a result of M. Kapovich and 
Leeb [13, Corollary 2.5] that given a finite cover $M_{1} \rightarrow M_{2}$ between closed 3-manifolds, $M_{1}$ admits a metric of nonpositive sectional curvature if and only if $M_{2}$ does.

Let $\bar{M}$ be a closed orientable 3-manifold without focal points. Since $\bar{M}$ is aspherical it is prime. By the geometrization it admits a geometric decomposition. By [8] or by Theorem 7.2, none of the geometric pieces are sol or nil. Also, if $\bar{M}$ is Seifert fibered then by Theorem A it is finitely covered by a product $S^{1} \times S_{g}$, where $S_{g}$ is a closed surface of genus $\geq 2$. All such manifolds obviously admit nonpositively curved metrics and hence so does $\bar{M}$. Thus we may assume that $\bar{M}$ is not Seifert fibered. If its geometric decomposition contains at least one hyperbolic piece then $\bar{M}$ admits a metric of nonpositive curvature by [15]. Thus we may assume that $\bar{M}$ is orientable, aspherical, not Seifert fibered, and its geometric decomposition has no nil or sol pieces. This means that $\bar{M}$ is a graph manifold and all its geometric pieces are modelled on $\mathbb{H}^{2} \times \mathbb{R}$.

Let us consider a single geometric piece $E$ of $\bar{M}$. It is Seifert fibered over a hyperbolizable 2-manifold with boundary. By Theorem 7.2 we can assume that all the boundary tori of $E$ (which are incompressible in $\bar{M}$ ) are flat and totally geodesic in $\bar{M}$. Let $E^{\prime} \rightarrow E$ be a finite normal cover which is topologically a product $S^{1} \times \Sigma$, where $\Sigma$ is a compact 2-manifold with boundary. Let $\gamma$ be a nontrivial element of $\pi_{1}(\bar{M})$ corresponding to the $S^{1}$ factor of $E^{\prime}$. Let $\pi: M \rightarrow \bar{M}$ be the universal cover of $\bar{M}$ and let $p: \tilde{E} \rightarrow E$ be the universal cover of $E$. Since the inclusion $E \hookrightarrow \bar{M}$ is $\pi_{1}$-injective we can think of $\tilde{E}$ as a subset of $M$.

Clearly, the preimages of the boundary tori of $E$ in $\tilde{E}$ belong to $A_{\gamma}$. This easily implies that the whole $\tilde{E}$ is contained in $A_{\gamma}$ by essentially the same argument as in Example 5.1. Indeed, since $\pi_{1}\left(E^{\prime}\right) \subset Z(\gamma)$ in $\pi_{1}(\bar{M}), d_{\gamma}$ descends from $\tilde{E}$ to a well-defined function $d_{\gamma}^{\prime}$ on $E^{\prime}$ and since $E^{\prime}$ is compact this function attains its maximum and minimum there. The whole boundary of $E^{\prime}$ belongs to the set of minima of $d_{\gamma}^{\prime}$ and since $d_{\gamma}$ has no critical points outside $A_{\gamma}$ by Lemma 2.1(4) we get that $d_{\gamma}^{\prime}$ is constant on $E^{\prime}$ and $d_{\gamma}$ is constant on $\tilde{E}$.

Therefore, by Proposition 7.3(2), $\tilde{E}$ isometrically splits as a product. More precisely, $\tilde{E}$ is isometric to $\mathbb{R} \times N^{2}$, where $N$ is the universal cover of $\Sigma$ equipped with some Riemannian metric with totally geodesic boundary. The isometric action of $\pi_{1}\left(E^{\prime}\right)=\pi_{1}\left(S^{1}\right) \times \pi_{1}(\Sigma)$ on $\mathbb{R} \times N$ preserves this splitting; furthermore, the action of the $\pi_{1}\left(S^{1}\right)$ factor preserves the $\mathbb{R}$-fibers of $\mathbb{R} \times N$ and the action of the $\pi_{1}(\Sigma)$ factor descends to the action on $N$ by deck transformations. Thus the metric of $N$ descends to a well defined Riemannian metric $g_{0}$ on $\Sigma$. Note that we do not claim that $E^{\prime}$ itself is isometric to a product.

Let us apply the uniformization procedure to the double of $\Sigma$. It produces a metric $g_{1}$ of constant negative curvature in the same conformal class as the original metric $g_{0}$. Since the uniformization procedure 
commutes with isometries, the boundary of $\Sigma$ remains totally geodesic. Also, the isometric action (by deck transformations) of $\pi_{1}(\Sigma)$ on $N$ with respect to $\tilde{g}_{0}$ remains isometric with respect to $\tilde{g}_{1}$. Therefore the action of $\pi_{1}(E)$ on $\tilde{E}$ is isometric with respect not only to the original product metric $\mathbb{R} \times\left(N, \tilde{g}_{0}\right)$ but also with respect to the new metric $\mathbb{R} \times\left(N, \tilde{g}_{1}\right)$, which is nonpositively curved. Notice that the conformal change of $g_{0}$ to $g_{1}$ may change the lengths of the boundary circles of $\Sigma$ but we can easily modify the metric $g_{1}$ to $g_{2}$ by rescaling and attaching tubular collars near the boundary so that the $g_{2}$ is still nonpositively curved and all the boundary circles have the same lengths as in $g_{0}$ and are still totally geodesic. Moreover, we can can easily arrange that $g_{2}$ is a product near the boundary. This yields a metric on $E$ that is nonpositively curved, $\partial E$ is totally geodesic and isometric to the original boundary, and the metric near $\partial E$ is flat. Doing it on all geometric pieces of $\bar{M}$ separately and gluing the resulting metrics together yields a metric of nonpositive curvature on $\bar{M}$.

q.e.d.

\section{Open problems}

The main open question concerning manifolds without conjugate points is Question 1.1, which we restate here.

Question 8.1. Does every closed Riemannian manifold without conjugate points admit a nonpositively curved metric? The same can be asked about manifolds without focal points.

While we suspect that this is likely false in general, it might be true in dimension 3 (cf. Theorem 7.1). The main problem is to understand which graph manifolds admit metrics without conjugate points. The corresponding question about nonpositively curved metrics is completely understood by the work of Buyalo and Kobelski [7].

The simplest test case to understand is the following

Question 8.2. Let $\Sigma=T^{2} \backslash D^{2}$. Let $\bar{M}_{1}=\bar{M}_{2}=\Sigma \times S^{1}$. Let $\bar{M}=\bar{M}_{1} \cup_{f} \bar{M}_{2}$, where $f$ is a self diffeomorphism of the boundary torus $T^{2}=\partial \bar{M}_{1}$ whose action on $\mathbb{Z}^{2}=\pi_{1}\left(T^{2}\right)$ is given by a matrix $A \in S L(2, \mathbb{Z})$ with $|\operatorname{trace}(A)|>2$. Then it is easy to see that $\bar{M}$ does not admit a metric of nonpositive curvature (say, by Theorem 7.2 and Proposition 7.3). Does $\bar{M}$ admit a metric without conjugate points?

QUestion 8.3. One of the natural classes containing CAT(0)-groups is the class of semi-hyperbolic groups. As was remarked in the introduction, it is known that in semi-hyperbolic groups all abelian subgroups are straight and all solvable subgroups are virtually abelian. It is therefore natural to pose the following weaker version of Question 1.1.

Is the fundamental group of a closed manifold without conjugate points semi-hyperbolic? 
Note however, that Theorem A does not hold for general semi-hyperbolic groups with the fundamental groups of unit tangent bundles to surfaces of genus $>1$ providing the simplest counterexamples.

Lastly, let us note that it is easy to see that if $\bar{M}$ has no focal points then $\pi_{1}(\bar{M})$ is semi-hyperbolic. Indeed, the no focal points condition implies that if $c_{1}(t), c_{2}(t)$ are geodesics in $M$ with $c_{1}(0)=c_{2}(0)$ then $d\left(c_{1}(t), c_{2}(t)\right)$ is monotone increasing for $t>0[\mathbf{1 7}$, Proposition 2]. This trivially implies that the canonical bicombing of $M$ by shortest geodesics satisfies the fellow traveller property and hence $\pi_{1}(\bar{M})$ is semi-hyperbolic.

QUESTION 8.4. Let $\bar{M}$ be a closed manifold without conjugate points and $\gamma \in \pi_{1}(\bar{M})$. Is it true that $Z(\gamma)$ is straight in $\pi_{1}(\bar{M})$ ? This is easily seen to be true if $\bar{M}$ has no focal points, because in this case $A_{\gamma}$ is convex in the universal cover $M$ and $A_{\gamma} / Z(\gamma)$ is compact. More generally it is known to be true for semi-hyperbolic groups [5].

\section{References}

[1] J. M. Alonso \& M. R. Bridson, Semihyperbolic groups, Proceedings of the London Mathematical Society, Third Series 70(1), 56-114, 1995, MR 1300841, Zbl 0823.20035.

[2] J. S. Birman, Braids, links, and mapping class groups, Annals of Mathematics Studies 82, Princeton University Press, Princeton, NJ, 1974, MR 0375281, Zbl 0305.57013.

[3] J. S. Birman, A. Lubotzky, \& J. McCarthy, Abelian and solvable subgroups of the mapping class groups, Duke Math. J. 50(4), 1107-1120, 1983, MR 0726319, Zbl 0551.57004.

[4] N. Brady, T. Riley, \& H. Short, The geometry of the word problem for finitely generated groups. Advanced Courses in Mathematics. CRM Barcelona. Birkhäuser Verlag, Basel, 2007. Papers from the Advanced Course held in Barcelona, July 5-15, 2005, MR 2281936, Zbl 1188.20026.

[5] M. R. Bridson \& A. Haefliger, Metric spaces of non-positive curvature, volume 319 of Grundlehren der Mathematischen Wissenschaften [Fundamental Principles of Mathematical Sciences]. Springer-Verlag, Berlin, 1999, MR 1744486 , Zbl 0988.53001.

[6] D. Burago, S. Ivanov, \& B. Kleiner, On the structure of the stable norm of periodic metrics, Mathematical Research Letters 4(6), 791-808, 1997, MR 1492121, Zbl 0898.53026.

[7] S. V. Buyalo \& V. L. Kobel'skiū, Geometrization of graph-manifolds, II. Isometric geometrization, St. Petersburg Math. J. 7(3), 387-404, 1996, MR 135349, $\mathrm{Zbl}$ 0855.57011.

[8] C. B. Croke \& V. Schroeder, The fundamental group of compact manifolds without conjugate points, Commentarii Mathematici Helvetici 61(1), 161-175, 1986, MR 0847526, Zbl 0608.53038 .

[9] C. J. Earle \& J. Eells, The diffeomorphism group of a compact Riemann surface, Bull. Amer. Math. Soc. 73, 557-559, 1967, MR 0212840, Zbl 0196.09402. 
[10] B. Farb, A. Lubotzky, \& Y. Minsky, Rank-1 phenomena for mapping class groups, Duke Math. J. 106(3), 581-597, 2001, MR 1813237, Zbl 1025.20023.

[11] R. Gulliver, On the variety of manifolds without conjugate points, Transactions of the American Mathematical Society 210, 185-201, Sept. 1975, MR 0383294, Zbl 0281.58008.

[12] N. V. Ivanov, Subgroups of Teichmüller modular groups, volume 115 of Translations of Mathematical Monographs. American Mathematical Society, Providence, RI, 1992. Translated from Russian by E.J.F. Primrose and revised by the author. MR 1195787, Zbl 0776.57001.

[13] M. Kapovich \& B. Leeb, Actions of discrete groups on nonpositively curved spaces, Mathematische Annalen 306(1), 341-352, 1996, MR 1411351, Zbl 0856.20024.

[14] N. Lebedeva, On the fundamental group of a compact space without conjugate points, PDMI preprint 5/2002, www.pdmi.ras.ru/preprint/2002/02-05.html, 2002.

[15] B. Leeb, 3-manifolds with(out) metrics of nonpositive curvature, Invent. Math. 122(2), 277-289, 1995, MR 1358977, Zbl 0840.53031.

[16] G. Mess, Unit tangent bundle subgroups of the mapping class groups, Preprint IHES/M/90/30, 1990.

[17] J. J. O'Sullivan, Riemannian manifolds without focal points, Journal of Differential Geometry 11(3), 321-333, 1976, MR 0431036, Zbl 0357.53026.

St. Petersburg Department of Steklov Mathematical Institute RAS

27 FONTANKA

191023 St. Petersburg, Russia

E-mail address: svivanov@pdmi.ras.ru

Department of Mathematics UNIVERSITY OF TORONTO

Toronto, Ontario M5S 2E4, Canada

E-mail address: vtk@math.toronto.edu 